\title{
Article \\ Photoredox Catalyzed Dealkylative Aromatic Halogen Substitution with Tertiary Amines
}

\author{
Dmitry L. Lipilin, Alexander E. Frumkin, Alexey Y. Tyurin, Vitalij V. Levin and Alexander D. Dilman *
}

Citation: Lipilin, D.L.; Frumkin, A.E.; Tyurin, A.Y.; Levin, V.V.; Dilman, A.D. Photoredox Catalyzed Dealkylative Aromatic Halogen Substitution with Tertiary Amines. Molecules 2021, 26, 3323. https://doi.org/10.3390/ molecules 26113323

Academic Editor: Viktor

O. Iaroshenko

Received: 29 April 2021

Accepted: 29 May 2021

Published: 1 June 2021

Publisher's Note: MDPI stays neutral with regard to jurisdictional claims in published maps and institutional affiliations.

Copyright: (c) 2021 by the authors. Licensee MDPI, Basel, Switzerland. This article is an open access article distributed under the terms and conditions of the Creative Commons Attribution (CC BY) license (https:/ / creativecommons.org/licenses/by/ $4.0 /)$.
N. D. Zelinsky Institute of Organic Chemistry, Leninsky Prosp. 47, 119991 Moscow, Russia; lipilin@ioc.ac.ru (D.L.L.); frumkin@ioc.ac.ru (A.E.F.); tyurin@ioc.ac.ru (A.Y.T.); levitavl@yandex.ru (V.V.L.)

* Correspondence: dilman@ioc.ac.ru

\begin{abstract}
A reaction of aromatic halides bearing electron-withdrawing groups with tertiary amines in the presence of an iridium catalyst under blue light irradiation is described. Products of the aromatic substitution of the halide by the dialkylamino fragment are obtained. The interaction of aryl radicals with tertiary amines to generate zwitterionic radical species is believed to be the key factor responsible for the reaction efficiency.
\end{abstract}

Keywords: aromatic substitution; tertiary amines; photoredox catalysis; radical reactions

\section{Introduction}

The substitution of halogen by heteroatom-centered nucleophiles in the aromatic ring is an important class of processes leading to numerous industrial products [1-4]. Since direct nucleophilic substitution proceeds through the loss of aromaticity, these reactions may require harsh conditions. Alternatively, aryl halides may be involved in the transition of metal catalyzed reactions mainly based on the application of complexes of palladium, nickel, or copper [5-9]. In the last decade, photoredox catalysis has emerged as a powerful tool for performing chemical reactions $[10,11]$ and it is also applied for aromatic substitution [12].

Several types of photoredox mediated substitution reactions were realized differing by the mode of action of the photocatalyst serving either as oxidant or reductant (Scheme 1). The oxidative pathway involves a single electron oxidation of the aromatic substrate by the light activated catalyst followed by nucleophilic attack and subsequent reduction [13-17]. This pathway works well for aromatics bearing electron donating groups. Another mode involves the application of strongly reducing catalysts, which can reduce the aromatic halide to generate aryl radical capable of interacting with a nucleophile with subsequent oxidation [18,19]. Reactions may also be performed via dual activation involving both photoredox and transition metal catalysis [20-23].

Oxidative pathway

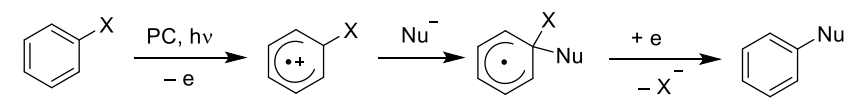

Reductive pathway

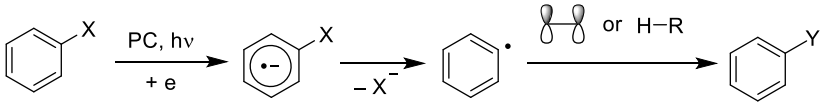

This work

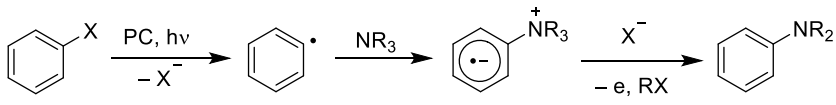

Scheme 1. Modes of photocatalytic substitution. 
The application of reductive photocatalysts toward generation of radicals is most appropriate for electron deficient substrates such as fluorinated or heteroaromatic compounds [24]. However, the resulting aryl radicals are typically trapped by $\pi$-systems (alkenes, alkynes, electron rich aromatics) or abstract a hydrogen atom from a suitable reagent [24-28].

Herein, we report a substitution of aromatic halides by dialkylamine group in reaction with tertiary amines. The reaction proceeds via the photoredox generation of the aryl radical, which interacts with the amine followed by loss of electron and elimination of one group from the ammonium fragment. Classical uncatalyzed nucleophilic substitution reactions of chlorine or heavier halogens with tertiary amines are known but proceed under thermal conditions [29-32], while such reactions of fluorides have not been described.

\section{Results and Discussion}

2,4-Dinitrofluorobenzene (1a) and triethylamine were selected as model compounds and their reaction was evaluated (Table 1). In dimethylsulfoxide, in the absence of photocatalyst or in the dark, there was no product formation at room temperature within $30 \mathrm{~h}$. Traces of amine 3a containing diethylamino fragment were observed when the reaction was heated at $50{ }^{\circ} \mathrm{C}$ for more than two days. Irradiation with blue LED at room temperature led to the $10 \%$ conversion (entry 3 ). Rewardingly, the use of photocatalysts gave noticeable improvement, with strongly reductive ones being more efficient. The best result was achieved using $f a c-\operatorname{Ir}(\mathrm{ppy})_{3}$ affording product $3 \mathrm{a}$ in $84 \%$ isolated yield (entry 8 ). Though incomplete conversion of 1a was observed, increase of the reaction time did not give the increase of the yield of 3a. Other solvents were evaluated but gave inferior results (entries 10-12).

Table 1. Optimization studies.

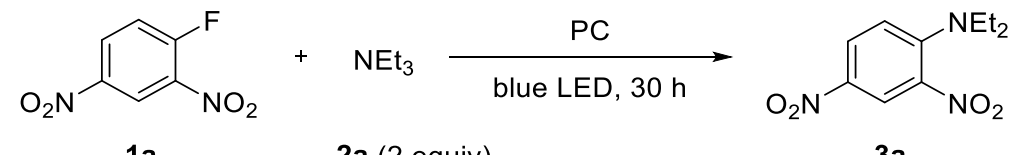

1a 2a (2 equiv) 3a

\begin{tabular}{|c|c|c|c|}
\hline Entry & $\mathrm{PC}^{1}$ & Solv. & Ratio 1a:3a \\
\hline $1^{2}$ & - & DMSO & 100:0 \\
\hline $2^{2,3}$ & - & DMSO & $95: 5$ \\
\hline 3 & - & DMSO & $90: 10$ \\
\hline 4 & $\mathrm{Ru}(\mathrm{phen})_{3}\left(\mathrm{PF}_{6}\right)_{2}(1 \%)$ & DMSO & $68: 32$ \\
\hline 5 & $4 \mathrm{CzIPN}(2 \%)$ & DMSO & $33: 67$ \\
\hline 6 & 3DPA2FBN (2\%) & DMSO & $28: 72$ \\
\hline 7 & $\operatorname{Ir}(\text { ppy })_{2}$ dtbbpy $\mathrm{PF}_{6}(0.5 \%)$ & DMSO & 29:71 \\
\hline 8 & $\operatorname{Ir}(\mathrm{ppy})_{3}(0.3 \%)$ & DMSO & $15: 85(84)^{4}$ \\
\hline 9 & $\operatorname{Ir}(\text { ppy })_{3}(0.3 \%), 1.1$ equiv of $\mathrm{NEt}_{3}$ & DMSO & $61: 39$ \\
\hline 10 & $\operatorname{Ir}(\mathrm{ppy})_{3}(0.3 \%)$ & DMF & $35: 65$ \\
\hline 11 & $\operatorname{Ir}(\mathrm{ppy})_{3}(0.3 \%)$ & $\mathrm{MeCN}$ & $57: 43$ \\
\hline 12 & $\operatorname{Ir}(\mathrm{ppy})_{3}(0.3 \%)$ & $\mathrm{CH}_{2} \mathrm{Cl}_{2}$ & $85: 15$ \\
\hline
\end{tabular}

${ }^{1}$ Abbreviations: 4CzIPN, 1,2,3,5-tetrakis(carbazol-9-yl)-4,6-dicyanobenzene; 3DPA2FBN, 2,4,6-tris(diphenylamino)3,5-difluorobenzonitrile. ${ }^{2}$ In the dark. ${ }^{3}$ At $50{ }^{\circ} \mathrm{C}$ for $60 \mathrm{~h} .{ }^{4}$ Isolated yield.

Under the above conditions, a series of tertiary amines were involved in the reaction with 2,4-dinitrofluorobenzene leading to products of the substitution of the fluoride by the dialkylamino group (Table 2). To reduce the amount of amine to 1.1 equiv, Hünig base (1 equiv) was added, which does not react with the substrate due to steric reasons but serves as a basic scavenger. Amines bearing non-identical substituents may afford various products. In the reaction of cyclic $N$-methylamines, the methyl group was selectively removed (products $3 \mathbf{c}-\mathbf{f}$ ). The reaction of 2-optimized cyanoethyldimethylamine unexpectedly furnished dimethyl-substituted amine $\mathbf{3} \mathbf{g}$ as a single product. The preferred removal of the 2-cyanoethyl compared to the methyl group may be associated with the presence of acidic proton at the $\alpha$-position of the nitrile, thereby favoring E2 elimination with the 
formation of acrylonitrile. In case of unsymmetrical alkyldimethylamines, the methyl group is cleaved preferentially, though traces of the products arising from the removal of another alkyl group were formed, and the portion of the latter products increases along with the expected efficiency of the nucleophilic substitution at carbon connected to nitrogen. These data allow to propose the following row of substituents with respect to increasing propensity of being detached from the amine: $\mathrm{Cy}<i-\operatorname{Pr}<$ primary alkyl $<\mathrm{Bn}<$ allyl.

Table 2. Reaction of fluoride 1a with amines.

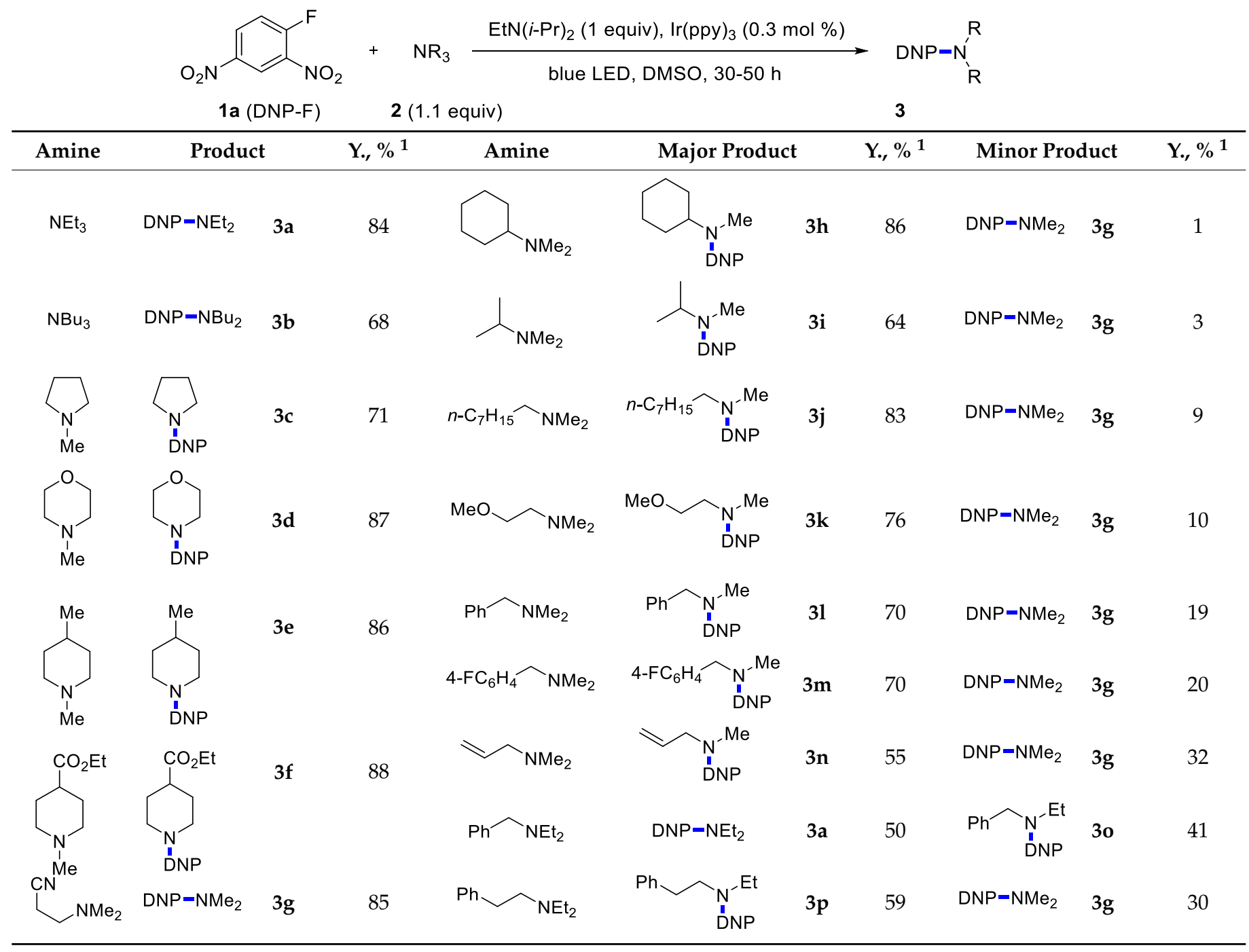

\footnotetext{
${ }^{1}$ Isolated yield.
}

Then, reactions of various aromatic substrates 1 with 2 equiv of triethylamine were evaluated (Scheme 2). The fluoride can be displaced starting from pentafluoropyridine and 4-thio-substituted tetrafluorinated pyridines $[33,34]$. The reaction worked well with a series of heteroaromatic chlorides. Derivatives of benzoxazole, oxadiazole, and diazines gave good yields. At the same time, 2-chlorobenzothiazole, 1-chloro-2,4,6-trinitrobenzene, 4-nitrofluorobenzene, 4-bromofluorobenzne, ethyl 4-fluorobenzoate were unreactive. 


$$
\underset{1}{\mathrm{Ar}-\mathrm{X}}+\underset{2 \text { equiv }}{\mathrm{NEt}_{3}} \stackrel{\operatorname{Ir}(\mathrm{ppy})_{3}(0.3 \mathrm{~mol} \%)}{\operatorname{blue~LED,~DMSO}, 15-40 \mathrm{~h}} \mathrm{Ar}-\mathrm{NEt}_{2}
$$

Fluoride substitution $(X=F)$<smiles>CCNc1ccc([N+](=O)[O-])cc1[N+](=O)[O-]</smiles><smiles>CCNc1c(F)c(F)nc(F)c1F</smiles><smiles>CCNc1nc(F)c(F)c(SC2CCCCC2)c1F</smiles><smiles>CCNc1nc(F)c(F)c(Sc2ccccc2)c1F</smiles><smiles>CCNc1nc(F)c(F)c(Sc2nc3ccccc3s2)c1F</smiles>

3a, $85 \%(40 \mathrm{~h})$

4a, $45 \%(30 \mathrm{~h})$

4b, $74 \%(20 h)$

4c, $51 \%(40 \mathrm{~h})$

4d, $65 \%(40 \mathrm{~h})$

$4 e, 56 \%(40 h)$

Chloride substitution $(\mathrm{X}=\mathrm{Cl})$<smiles>CCNc1nc2ccccc2o1</smiles>

4f, $87 \%(30 \mathrm{~h})$<smiles>CCNc1nc2cc(C)ccc2o1</smiles>

4g, $84 \%(20 h)$<smiles>CCNc1nnc(-c2ccccc2)s1</smiles>

4h, $77 \%$ (30 h)<smiles>CCNc1ccnc(Cl)n1</smiles>

$4 i, 72 \%(30 h)$<smiles>CCNc1ncccc1[N+](=O)[O-]</smiles>

4n, $97 \%(15 \mathrm{~h})$<smiles>CCNc1nccnc1Cl</smiles>

4j, $83 \%(40 h)$<smiles>CCNc1ncc(Br)cc1[N+](=O)[O-]</smiles>

40, $96 \%(15 h)$

Bromide substitution $(X=\mathrm{Br}$ )<smiles>CCNc1nc(C(C)C)no1</smiles>

$4 p, 86 \%(15 h)$

Scheme 2. Reaction of aryl halides with $\mathrm{NEt}_{3}$. Isolated yields are shown, reaction times are in parenthesis. ${ }^{1}$ Performed in $\mathrm{CH}_{2} \mathrm{Cl}_{2}$.

The proposed mechanism is shown in Scheme 3. Starting aryl halide is reduced by the photoexcited iridium complex with elimination of the halide anion and generation of the aryl radical. The radical interacts with tertiary amine leading to zwitterionic species $\mathbf{A}$, which is converted into the product 3 along with regeneration of the $\operatorname{Ir}(\mathrm{III})$ complex. The transformation of intermediate $\mathbf{A}$ into the product involves single electron oxidation of the aromatic $\pi$-system and removal of the alkyl group from nitrogen with the aid of the amine (Scheme 3, right). The detachment of the alkyl group may be realized either via nucleophilic substitution or through E2 elimination mechanism, especially if the group has acidic hydrogen at the $\beta$-position. The order of oxidation and alkyl group removal may depend on the nature of substituents in the aromatic ring and at the nitrogen atom. The radical character of the process was supported by an experiment with a radical trap. Thus, when the reaction of 2,4-dinitrofluorobenzene with triethylamine were carried out in the presence of TEMPO (1.5 equiv) under standard conditions, no product was formed, leaving the starting arylfluoride unconsumed. 


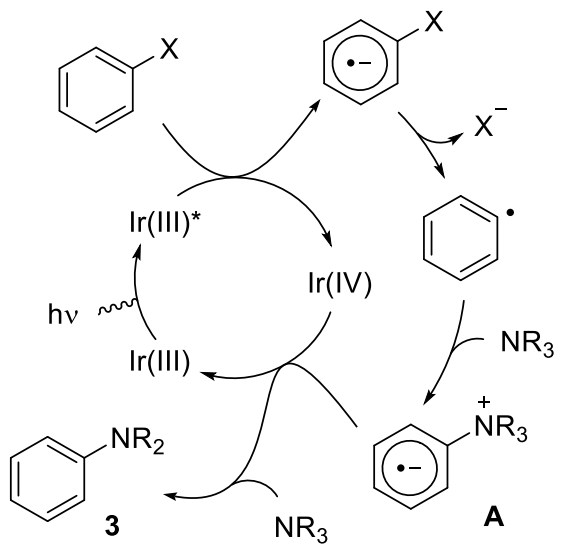

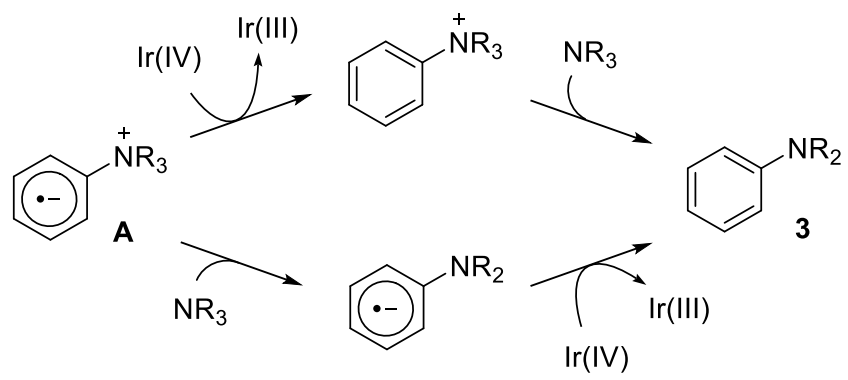

Scheme 3. Proposed mechanism.

The redox potential of 2,4-dinitrofluorobenzene (1a) in DMSO was measured by cyclic voltammetry affording a value of $-0.93 \mathrm{~V}$ (vs. SCE) (see Supplementary Materials for $\mathrm{CV}$ curves). The photoexcited $f a c-\operatorname{Ir}(\mathrm{ppy})_{3}$ has the potential of $-1.73 \mathrm{~V}$ (from $\operatorname{Ir}(\mathrm{III})^{*}$ to $\operatorname{Ir}(\mathrm{IV})$ ) [10], thereby suggesting facile single electron reduction of 1a. It should also be pointed out that the photoexcited catalyst may be reductively quenched by tertiary amine generating $\operatorname{Ir}(\mathrm{II})$, which has even stronger reducing power (for $\operatorname{Ir}(\mathrm{II}) / \operatorname{Ir}(\mathrm{III}),-2.19 \mathrm{~V}$ [10]). The use of iridium photocatalysts for the generation of aryl radicals by reductive cleavage of the C-F bond in aromatic aryl fluorides was described [24].

To gain insight into the alkyl detachment step from intermediate $\mathbf{A}$, a photoreaction of pentafluoropyridine with two equivalents of $N$-methylpyrrolidine was evaluated (Scheme 4). The reaction was performed in acetonitrile, and after completion the mixture was directly analyzed by ${ }^{1} \mathrm{H},{ }^{13} \mathrm{C},{ }^{19} \mathrm{~F}$ NMR, and no alkyl fluorides were observed. The acetonitrile was evaporated and the residue was washed to remove all non-ionic organic compounds. When the residue was analyzed by NMR spectroscopy in DMSO- $\mathrm{d}_{6}$, $N, N$-dimethylpyrrolidinium cation was observed (the reference ammonium cation was formed by interaction of $N$-methylpyrrolidine with methyl iodide). The combined methyl tert-butyl ether phases were analyzed by GC-MS demonstrating that tetrafluorinated 4pyrrolydinopyridine is a single substitution product.

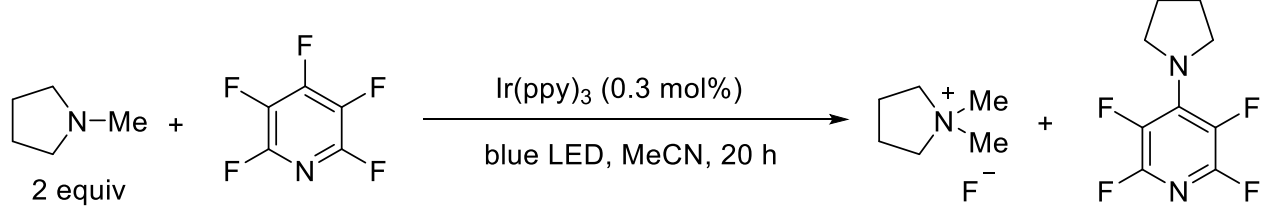

Scheme 4. Formation of ammonium salt.

To support the hypothesis of the formation of species $\mathbf{A}$, the free energies of the interaction of aryl radicals with trimethylamine were derived by means of quantum chemical calculations. Energies were calculated at M06-2X/6-31+G(d) [35], and for the stationary points, energies were calculated by CPCM method in DMSO solution (Figure 1). In case of perfluorinated pyridinyl and benzoxazolyl radicals, in the resulting complexes A1 and A2, the amine nitrogen is located out of plane of the heteroaromatic ring having relatively long C,N bond of $1.595 \AA$ and $1.559 \AA$, respectively. Such a geometry can be considered as a weak Meisenheimer complex between the amine and the aromatic system. On the other hand, for the complexes originated from 2,4-dinitrophenyl and 3-nitropyridinyl radicals, the amine fragment is located within the plane of the aromatic ring, with the $\mathrm{C}, \mathrm{N}$ bonds in A3 and A4 being equal to $1.507 \AA$ and $1.500 \AA$, respectively. Of special note, for the latter 
complexes, the free energies of their formation are notably more negative compared to those of $\mathbf{A} 1$ and $\mathbf{A} 2$.

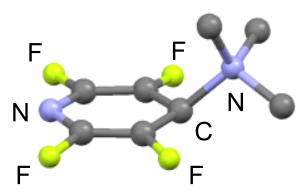

A1

$\mathrm{C...NMe}_{3}(\AA) \quad 1.595$

$\Delta \mathrm{G}(\mathrm{kcal} / \mathrm{mol}) \quad-6.8$

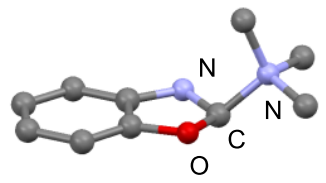

A2

1.559

$-7.3$

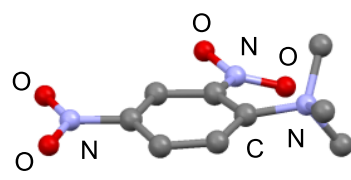

A3

1.507

$-18.7$

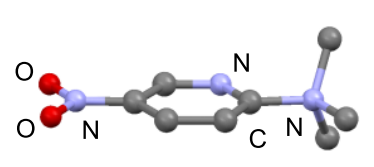

A4

1.500

$-28.3$

Figure 1. Calculated structures of complexes $\mathbf{A}$ formed upon interaction of aryl radicals with $\mathrm{NMe}_{3}$ (UM06-2X/6-31+G(d), DMSO as solvent). Hydrogen atoms are omitted. Bond length between the carbon atom of the aromatic ring and the nitrogen and free energies of complex formation are shown.

\section{Materials and Methods}

\subsection{General Information}

All reactions were performed under an argon atmosphere. DMSO was distilled from $\mathrm{CaH}_{2}$ and stored over MS $4 \AA$. Column chromatography was carried out employing silica gel (230-400 mesh). High resolution mass-spectra (HRMS) were measured using electrospray ionization (ESI) and a time-of-flight (TOF) mass analyzer (Bruker MicrOTOF II, Bruker, Billerica, USA). The measurements were done in a positive-ion mode (interface capillary voltage $-4500 \mathrm{~V}$ ) or in a negative-ion mode ( $3200 \mathrm{~V})$; the mass ranged from $\mathrm{m} / \mathrm{z}$ 50 to $\mathrm{m} / \mathrm{z}$ 3000. For irradiation, a strip of $455 \mathrm{~nm}$ light-emitting diodes (SMD 2835-120 LED $1 \mathrm{M}$ Blue, $12 \mathrm{~V}, 24 \mathrm{~W} / \mathrm{m}$; $70 \mathrm{~cm}$ strip length) was used. The distance between the reaction vessel and diodes was about $30 \mathrm{~mm}$, and the reaction vial was cooled with a fan.

4-(Cyclohexylthio)-2,3,5,6-tetrafluoropyridine [34] and 2,3,5,6-tetrafluoro-4(phenylthio)pyridine [36] were obtained according to literature procedures.

2-[(2,3,5,6-Tetrafluoropyridin-4-yl)sulfanyl]-1,3-benzothiazole. Potassium carbonate (1.74 g, $12.8 \mathrm{mmol})$ was added to a solution of 2-mercaptobenzothiazole $(2.1 \mathrm{~g}, 12.6 \mathrm{mmol})$ in DMF $(15 \mathrm{~mL})$. The mixture was stirred for $15 \mathrm{~min}$, then added pentafluoropyridine ( $2.3 \mathrm{~g}$, $13.8 \mathrm{mmol}$ ), and the mixture was stirred for one hour at room temperature. The mixture was poured into water $(100 \mathrm{~mL})$, the precipitate was filtered, washed with water, and dried in air. The obtained material was purified vie short silica gel column eluting with dichloromethane. Yield $3.2 \mathrm{~g}(80 \%)$. Colorless solid. Mp 69-71 ${ }^{\circ} \mathrm{C}(\mathrm{EtOH}) .{ }^{1} \mathrm{H}$ NMR $\left(300 \mathrm{MHz}, \mathrm{DMSO}_{\mathrm{d}}\right) \delta 8.10(\mathrm{~d}, J=7.4 \mathrm{~Hz}, 1 \mathrm{H}), 7.92(\mathrm{~d}, J=7.6 \mathrm{~Hz}, 1 \mathrm{H}), 7.47-7.53(\mathrm{~m}$, 2H). ${ }^{13} \mathrm{C}$ NMR $(75 \mathrm{MHz}$, DMSO-d 6 ) $\delta 149.4 .7,152.6,143.4(\mathrm{dm}, J=239 \mathrm{~Hz}), 142.5(\mathrm{dm}$ $J=258 \mathrm{~Hz}), 136.3,127.2,126.1,125.2(\mathrm{~m}), 122.6,122.5 .{ }^{19} \mathrm{~F}$ NMR $\left(282 \mathrm{MHz}, \mathrm{DMSO}-\mathrm{d}_{6}\right)$ $\delta-90.8(\mathrm{~d}, J=7 \mathrm{~Hz}),-134.1(\mathrm{~d}, J=7 \mathrm{~Hz})$. HRMS (ESI): calcd for $\mathrm{C}_{12} \mathrm{H}_{5} \mathrm{~F}_{4} \mathrm{~N}_{2} \mathrm{~S}_{2}, 316.9825$ $[\mathrm{M}+\mathrm{H}]$; found, 316.9814 .

\subsection{Reactions of Aryl Halides with Amines}

3.2.1. Reaction of 2,4-Dinitrofluorobenzene with Tertiary Amines. Synthesis of Amines 3. General Procedure 1 (GP 1)

$f a c-\operatorname{Ir}(\mathrm{ppy})_{3}(2 \mathrm{mg}, 0.003 \mathrm{mmol}), \mathrm{EtN}(i-\operatorname{Pr})_{2}(174 \mu \mathrm{L}, 1 \mathrm{mmol})$ and amine $(1.1 \mathrm{mmol})$ were added to a solution of 2,4-dinitrofluorobenezene (186 mg, $1 \mathrm{mmol})$ in DMSO $(2 \mathrm{~mL})$. The mixture was irradiated at room temperature with blue LED [for $3 \mathbf{c}, \mathbf{g}, \mathbf{j}, \mathbf{n}, 30 \mathrm{~h}$; for $\mathbf{3 a}, \mathbf{b}, \mathbf{d}-\mathbf{f}, \mathbf{h}, \mathbf{i}, \mathbf{k}, \mathbf{m}, 40 \mathrm{~h}$; for $3 \mathbf{l}, \mathbf{o}, \mathbf{p}, 50 \mathrm{~h}]$. For the work-up, the mixture was poured into $2 \%$ aqueous hydrochloric acid $(20 \mathrm{~mL})$ and extracted with EtOAc $(4 \times 15 \mathrm{~mL})$. The combined organic phases were washed with water $(10 \mathrm{~mL})$, a $5 \%$ solution of potassium carbonate $(10 \mathrm{~mL})$, brine $(10 \mathrm{~mL})$, and dried over $\mathrm{Na}_{2} \mathrm{SO}_{4}$. The solvent was evaporated, and the residue was purified by column chromatography eluting with dichloromethane. 
3.2.2. Reactions of Aryl Halides with Tertiary Amines. Synthesis of Amines 4. General Procedure 2 (GP 2)

fac-Ir(ppy) $3(2 \mathrm{mg}, 0.003 \mathrm{mmol})$ and $\mathrm{NEt}_{3}(282 \mu \mathrm{L}, 2 \mathrm{mmol})$ were added to a solution of aromatic halide $(1 \mathrm{mmol})$ in DMSO $(2 \mathrm{~mL})$. The mixture was irradiated at room temperature with blue LED for 15-40 h (the reaction time is shown in Scheme 3). The work-up is the same as in General Procedure 1.

N,N-Diethyl-2,4-dinitroaniline (3a) [37]. According to GP 1; yield $201 \mathrm{mg}$ (84\%). According to GP 2 from $\mathrm{BnNEt}_{2}$, yield $120 \mathrm{mg}$ (50\%); from $\mathrm{PhCH}_{2} \mathrm{CH}_{2} \mathrm{NEt}_{2}$, yield $72 \mathrm{mg}(30 \%)$; from $\mathrm{NEt}_{3}$, yield $203 \mathrm{mg}(85 \%)$. Yellow solid. Mp 75-77 ${ }^{\circ} \mathrm{C} .{ }^{1} \mathrm{H} \mathrm{NMR}\left(300 \mathrm{MHz}, \mathrm{CDCl}_{3}\right)$ $\delta 8.64(\mathrm{~d}, J=2.8 \mathrm{~Hz}, 1 \mathrm{H}), 8.21(\mathrm{dd}, J=9.6 \mathrm{~Hz}, 2.8 \mathrm{~Hz} 1 \mathrm{H}), 7.08(\mathrm{~d}, J=9.6 \mathrm{~Hz}, 1 \mathrm{H}), 3.38$ $(\mathrm{q}, J=7.2 \mathrm{~Hz}, 4 \mathrm{H}), 1.26(\mathrm{t}, J=7.2 \mathrm{~Hz}, 6 \mathrm{H}) .{ }^{13} \mathrm{C} \mathrm{NMR}\left(75 \mathrm{MHz}, \mathrm{CDCl}_{3}\right) \delta 148.0,137.2,136.4$, $127.5,123.8,118.4,46.3,12.3$.

N,N-Dibutyl-2,4-dinitroaniline (3b) [38]. Yield $201 \mathrm{mg}(68 \%)$. Orange liquid. ${ }^{1} \mathrm{H}$ $\operatorname{NMR}\left(300 \mathrm{MHz}, \mathrm{CDCl}_{3}\right) \delta 8.65(\mathrm{~d}, J=2.8 \mathrm{~Hz}, 1 \mathrm{H}), 8.19(\mathrm{dd}, J=9.5 \mathrm{~Hz}, 2.8 \mathrm{~Hz} 1 \mathrm{H})$, $7.08(\mathrm{~d}, J=9.5 \mathrm{~Hz}, 1 \mathrm{H}), 3.28(\mathrm{t}, J=7.3 \mathrm{~Hz}, 2 \mathrm{H}), 1.59(\mathrm{~m}, 2 \mathrm{H}), 1.31(\mathrm{~m}, 2 \mathrm{H}), 0.93(\mathrm{t}, J=7.7 \mathrm{~Hz}$, $3 \mathrm{H}) .{ }^{13} \mathrm{C} \mathrm{NMR}\left(75 \mathrm{MHz}, \mathrm{CDCl}_{3}\right) \delta 148.7,137.7,136.8,127.6,124.0,118.8,52.0,29.4,20.0$, 13.7.

1-(2,4-Dinitrophenyl)pyrrolidine (3c) [39]. Yield $168 \mathrm{mg}$ (71\%). Yellow solid. Mp 97-98 ${ }^{\circ} \mathrm{C} .{ }^{1} \mathrm{H}$ NMR $\left(300 \mathrm{MHz}, \mathrm{CDCl}_{3}\right) \delta 8.68(\mathrm{~d}, J=2.6 \mathrm{~Hz}, 1 \mathrm{H}), 8.21(\mathrm{dd}, J=9.5 \mathrm{~Hz}$, $2.6 \mathrm{~Hz} 1 \mathrm{H}), 6.92(\mathrm{~d}, J=9.5 \mathrm{~Hz}, 1 \mathrm{H}), 3.37(\mathrm{~m}, 4 \mathrm{H}), 1.26(\mathrm{~m}, 4 \mathrm{H}) .{ }^{13} \mathrm{C} \mathrm{NMR}\left(75 \mathrm{MHz}, \mathrm{CDCl}_{3}\right)$ $\delta 145.6,135.3,134.8,127.5,123.8,115.6,51.1,25.6$.

4-(2,4-Dinitrophenyl)morpholine (3d) [40]. Yield $220 \mathrm{mg}$ (87\%). Yellow solid. Mp 116-118 ${ }^{\circ} \mathrm{C} .{ }^{1} \mathrm{H}$ NMR $\left(300 \mathrm{MHz} \mathrm{CDCl}_{3}\right) \delta 8.70(\mathrm{~d}, J=2.8 \mathrm{~Hz}, 1 \mathrm{H}), 8.29(\mathrm{dd}, J=9.4 \mathrm{~Hz}$, $2.8 \mathrm{~Hz} 1 \mathrm{H}), 7.13(\mathrm{~d}, J=9.4 \mathrm{~Hz}, 1 \mathrm{H}), 3.88(\mathrm{~m}, 4 \mathrm{H}), 3.29(\mathrm{~m}, 4 \mathrm{H}) .{ }^{13} \mathrm{C} \mathrm{NMR}\left(75 \mathrm{MHz}, \mathrm{CDCl}_{3}\right)$ $\delta 149.3,139.9,138.6,128.4,123.6,119.2,66.2,50.9$.

1-(2,4-Dinitrophenyl)-4-methylpiperidine (3e) [41]. Yield $228 \mathrm{mg}(86 \%)$. Yellow solid. Mp 84-86 ${ }^{\circ} \mathrm{C} .{ }^{1} \mathrm{H}$ NMR $\left(300 \mathrm{MHz}, \mathrm{CDCl}_{3}\right) \delta 8.70(\mathrm{~d}, J=2.7 \mathrm{~Hz}, 1 \mathrm{H}), 8.23(\mathrm{dd}, J=9.4 \mathrm{~Hz}, 2.7 \mathrm{~Hz}$ $1 \mathrm{H}), 7.09(\mathrm{~d}, J=9.4 \mathrm{~Hz}, 1 \mathrm{H}), 3.45(\mathrm{~m}, 2 \mathrm{H}), 3.11(\mathrm{~m}, 2 \mathrm{H}), 1.76-1.82(\mathrm{~m}, 3 \mathrm{H}), 1.39(\mathrm{~m}, 2 \mathrm{H}), 1.03$ $(\mathrm{d}, J=6.4 \mathrm{~Hz}, 3 \mathrm{H}) .{ }^{13} \mathrm{C} \mathrm{NMR}\left(75 \mathrm{MHz}, \mathrm{CDCl}_{3}\right) \delta 149.7,137.3,137.2,128.0,123.9,119.2,51.2$, 33.6, 30.2, 21.6 .

Ethyl 1-(2,4-dinitrophenyl)piperidine-4-carboxylate (3f). Yield $284 \mathrm{mg}$ (88\%). Yellow solid. Mp 94-96 ${ }^{\circ} \mathrm{C}(\mathrm{EtOH}) .{ }^{1} \mathrm{H}$ NMR $\left(300 \mathrm{MHz}, \mathrm{CDCl}_{3}\right) \delta 8.72(\mathrm{~d}, J=2.6 \mathrm{~Hz}, 1 \mathrm{H}), 8.26$ (dd, $J=9.3 \mathrm{~Hz}, 2.6 \mathrm{~Hz} 1 \mathrm{H}), 7.12(\mathrm{~d}, J=9.3 \mathrm{~Hz}, 1 \mathrm{H}), 4.21(\mathrm{q}, J=7.1 \mathrm{~Hz}, 2 \mathrm{H}), 3.48(\mathrm{~m}, 2 \mathrm{H}), 3.18(\mathrm{~m}$, $2 \mathrm{H}), 2.63(\mathrm{~m}, 1 \mathrm{H}), 2.06-2.61(\mathrm{~m}, 4 \mathrm{H}), 1.97(\mathrm{t}, J=7.1 \mathrm{~Hz}, 3 \mathrm{H}) .{ }^{13} \mathrm{C} \mathrm{NMR}\left(75 \mathrm{MHz}, \mathrm{CDCl}_{3}\right) \delta$ $173.8,149.6,138.1,137.9,128.2,123.8,119.5,60.8,50.2,39.9,27.6,14.2$. HRMS (ESI): calcd for $\mathrm{C}_{14} \mathrm{H}_{18} \mathrm{~N}_{3} \mathrm{O}_{6}[\mathrm{M}+\mathrm{H}], 324.1190$; found, 324.1188.

$\mathrm{N}, \mathrm{N}$-Dimethyl-2,4-dinitroaniline (3g) [42]. Yield $179 \mathrm{mg}(85 \%)$ from $\mathrm{Me}_{2} \mathrm{NCH}_{2} \mathrm{CH}_{2} \mathrm{CN}$. Yield $68 \mathrm{mg}$ (32\%) from AllylNMe . Yield $42 \mathrm{mg}(20 \%)$ from 4-FPhCH $\mathrm{NMe}_{2}$. Yield $2 \mathrm{mg}$ (1\%) from CyclohexylNMe 2 . Yield $19 \mathrm{mg}(9 \%)$ from $\mathrm{C}_{8} \mathrm{H}_{17} \mathrm{NMe}_{2}$. Yield $40 \mathrm{mg}$ (19\%) from $\mathrm{BnNMe}_{2}$. Yield $21 \mathrm{mg}(10 \%)$ from $\mathrm{MeOCH}_{2} \mathrm{CH}_{2} \mathrm{NMe}_{2}$. Yield $6 \mathrm{mg}$ (3\%) from $i-\mathrm{PrNMe}_{2}$. Yellow solid. Mp 84-86 ${ }^{\circ} \mathrm{C} .{ }^{1} \mathrm{H}$ NMR $\left(300 \mathrm{MHz}, \mathrm{CDCl}_{3}\right) \delta 8.60(\mathrm{~d}, J=2.7 \mathrm{~Hz}, 1 \mathrm{H}), 8.15$ (dd, $J=9.5 \mathrm{~Hz}, 2.7 \mathrm{~Hz} 1 \mathrm{H}), 7.01(\mathrm{~d}, J=9.5 \mathrm{~Hz}, 1 \mathrm{H}), 3.06(\mathrm{~s}, 6 \mathrm{H}) .{ }^{13} \mathrm{C} \mathrm{NMR}\left(75 \mathrm{MHz}, \mathrm{CDCl}_{3}\right) \delta$ $149.2,136.2,135.5,127.7,124.1,116.7,42.4$.

N-Cyclohexyl-N-methyl-2,4-dinitroaniline (3h) [43]. Yield $240 \mathrm{mg}$ (86\%). Yellow solid. Mp 115-118 ${ }^{\circ} \mathrm{C} .{ }^{1} \mathrm{H}$ NMR $\left(300 \mathrm{MHz}, \mathrm{CDCl}_{3}\right) \delta 8.64(\mathrm{~d}, J=2.7 \mathrm{~Hz}, 1 \mathrm{H}), 8.16(\mathrm{dd}, J=9.6 \mathrm{~Hz}$, $2.7 \mathrm{~Hz} 1 \mathrm{H}), 7.08(\mathrm{~d}, J=9.6 \mathrm{~Hz}, 1 \mathrm{H}), 3.57(\mathrm{~m}, 1 \mathrm{H}), 2.77(\mathrm{~s}, 3 \mathrm{H}), 1.91(\mathrm{~m}, 4 \mathrm{H}), 1.21-1.76(\mathrm{~m}$, $6 \mathrm{H}) .{ }^{13} \mathrm{C} \mathrm{NMR}\left(75 \mathrm{MHz}, \mathrm{CDCl}_{3}\right) \delta 149.4,136.3,136.1,127.5,124.3,117.9,62.1,34.6,29.8$, 25.6, 25.4 .

N-Methyl-N-(1-methylethyl)-2,4-dinitroaniline (3i). Yield $153 \mathrm{mg}$ (64\%). Yellow solid. $\mathrm{Mp} 73-75{ }^{\circ} \mathrm{C}(\mathrm{EtOH}) .{ }^{1} \mathrm{H} \mathrm{NMR}\left(300 \mathrm{MHz}, \mathrm{CDCl}_{3}\right) \delta 8.66(\mathrm{~d}, J=2.7 \mathrm{~Hz}, 1 \mathrm{H}), 8.19$ (dd, $J=9.6 \mathrm{~Hz}, 2.7 \mathrm{~Hz} 1 \mathrm{H}), 7.09(\mathrm{~d}, J=9.6 \mathrm{~Hz}, 1 \mathrm{H}), 4.03$ (hept, $J=6.6 \mathrm{~Hz}, 1 \mathrm{H}), 2.75(\mathrm{~s}, 3 \mathrm{H}), 1.33$ $(\mathrm{d}, J=6.6 \mathrm{~Hz}, 6 \mathrm{H}) .{ }^{13} \mathrm{C} \mathrm{NMR}\left(75 \mathrm{MHz}, \mathrm{CDCl}_{3}\right) \delta 149.3,136.4,136.3,127.7,124.3,117.7,53.3$, 33.0, 19.4. HRMS (ESI): calcd for $\mathrm{C}_{10} \mathrm{H}_{14} \mathrm{~N}_{3} \mathrm{O}_{4}[\mathrm{M}+\mathrm{H}], 240.0979$; found, 240.0975. 
N-Methyl-2,4-dinitro-N-octylaniline (3j). Yield $256 \mathrm{mg}$ (83\%). Orange liquid. ${ }^{1} \mathrm{H}$ NMR $\left(300 \mathrm{MHz}, \mathrm{CDCl}_{3}\right) \delta 8.65(\mathrm{~d}, J=2.7 \mathrm{~Hz}, 1 \mathrm{H}), 8.17(\mathrm{dd}, J=9.5 \mathrm{~Hz}, 2.7 \mathrm{~Hz} 1 \mathrm{H})$, $7.05(\mathrm{~d}, J=9.5 \mathrm{~Hz}, 1 \mathrm{H}), 3.38(\mathrm{t}, J=7.6 \mathrm{~Hz}, 2 \mathrm{H}), 2.93(\mathrm{~s}, 3 \mathrm{H}), 1.31(\mathrm{~m}, 2 \mathrm{H}), 1.26-1.30(\mathrm{~m}, 10 \mathrm{H})$, $0.87(\mathrm{t}, J=7.0 \mathrm{~Hz}, 3 \mathrm{H}) .{ }^{13} \mathrm{C}$ NMR $\left(75 \mathrm{MHz}, \mathrm{CDCl}_{3}\right) \delta 148.8,136.2,136.0,127.6,124.2,117.2$, 54.2, 40.5, 31.7, 29.2, 29.1, 26.9, 26.6, 22.6, 14.0. HRMS (ESI): calcd for $\mathrm{C}_{15} \mathrm{H}_{24} \mathrm{~N}_{3} \mathrm{O}_{4}[\mathrm{M}+\mathrm{H}]$ 310.1761; found, 310.1758 .

$\mathrm{N}$-(2-Methoxyethyl)-N-methyl-2,4-dinitroaniline (3k). Yield $194 \mathrm{mg}$ (76\%). Orange liquid. ${ }^{1} \mathrm{H}$ NMR $\left(300 \mathrm{MHz}, \mathrm{CDCl}_{3}\right) \delta 8.62(\mathrm{~d}, J=2.6 \mathrm{~Hz}, 1 \mathrm{H}), 8.17(\mathrm{dd}, J=9.5 \mathrm{~Hz}, 2.6 \mathrm{~Hz} 1 \mathrm{H}), 7.19$ $(\mathrm{d}, J=9.5 \mathrm{~Hz}, 1 \mathrm{H}), 3.65(\mathrm{t}, J=5.1 \mathrm{~Hz}, 2 \mathrm{H}), 3.58(\mathrm{t}, J=5.1 \mathrm{~Hz}, 2 \mathrm{H}), 3.35(\mathrm{~s}, 3 \mathrm{H}), 3.00(\mathrm{~s}, 3 \mathrm{H})$. ${ }^{13} \mathrm{C}$ NMR $\left(75 \mathrm{MHz}, \mathrm{CDCl}_{3}\right.$ ) $\delta 149.2,136.7,136.4,127.5,123.9,118.3,69.5,59.1,54.0,40.3$. HRMS (ESI): calcd for $\mathrm{C}_{10} \mathrm{H}_{14} \mathrm{~N}_{3} \mathrm{O}_{5}$ [M + H], 256.0928, found, 256.0926 .

$\mathrm{N}$-Benzyl-N-methyl-2,4-dinitroaniline (31) [44]. Yield $201 \mathrm{mg}(70 \%)$. Yellow solid. Mp 143-145 ${ }^{\circ} \mathrm{C} .{ }^{1} \mathrm{H}$ NMR $\left(300 \mathrm{MHz}, \mathrm{CDCl}_{3}\right) \delta 8.70(\mathrm{~d}, J=2.6 \mathrm{~Hz}, 1 \mathrm{H}), 8.16(\mathrm{dd}, J=9.5 \mathrm{~Hz}$, $2.6 \mathrm{~Hz} 1 \mathrm{H}), 7.22-7.39(\mathrm{~m}, 5 \mathrm{H}), 7.05(\mathrm{~d}, J=9.5 \mathrm{~Hz}, 1 \mathrm{H}), 4.66(\mathrm{~s}, 2 \mathrm{H}), 2.97(\mathrm{~s}, 3 \mathrm{H}) .{ }^{13} \mathrm{C} \mathrm{NMR}$ $\left(75 \mathrm{MHz}, \mathrm{CDCl}_{3}\right) \delta 149.1,137.0,136.4,134.9,129.2,128.1,127.7,126.9,124.0,118.0,57.8$, 41.0 .

$\mathrm{N}$-(4-Fluorobenzyl)-N-methyl-2,4-dinitroaniline (3m). Yield $214 \mathrm{mg}(70 \%)$. Orange liquid. ${ }^{1} \mathrm{H} \mathrm{NMR}\left(300 \mathrm{MHz}, \mathrm{CDCl}_{3}\right) \delta 8.68(\mathrm{~d}, J=2.7 \mathrm{~Hz}, 1 \mathrm{H}), 8.17(\mathrm{dd}, J=9.5 \mathrm{~Hz}, 2.7 \mathrm{~Hz} 1 \mathrm{H}), 7.22$ $(\mathrm{m}, 2 \mathrm{H}), 7.03-7.10(\mathrm{~m}, 3 \mathrm{H}), 4.61(\mathrm{~s}, 2 \mathrm{H}), 2.95(\mathrm{~s}, 3 \mathrm{H}) .{ }^{13} \mathrm{C} \mathrm{NMR}\left(75 \mathrm{MHz}, \mathrm{CDCl}_{3}\right) \delta 162.4$ $(\mathrm{d}, J=245.5 \mathrm{~Hz}), 149.0,137.3,136.7,130.7 .8,128.9(\mathrm{~d}, J=22.7 \mathrm{~Hz}), 127.8,123.9,118.1,116.1$ $(\mathrm{d}, J=7.6 \mathrm{~Hz}), 57.2,40.9 .{ }^{19} \mathrm{~F}$ NMR $\left(282 \mathrm{MHz}, \mathrm{CDCl}_{3}\right) \delta-114.54$. HRMS (ESI): calcd for $\mathrm{C}_{14} \mathrm{H}_{12} \mathrm{FN}_{3} \mathrm{O}_{4} \mathrm{Na}$ [M + Na], 328.0704; found, 328.0693.

N-Methyl-2,4-dinitro-N-prop-2-en-1-ylaniline (3n) [45]. Yield $130 \mathrm{mg}$ (55\%). Yellow solid. Mp 60-62 ${ }^{\circ} \mathrm{C} .{ }^{1} \mathrm{H}$ NMR $\left(300 \mathrm{MHz}, \mathrm{CDCl}_{3}\right) \delta 8.65(\mathrm{~d}, J=2.7 \mathrm{~Hz}, 1 \mathrm{H}), 8.18(\mathrm{dd}, J=9.5 \mathrm{~Hz}$, $2.7 \mathrm{~Hz} 1 \mathrm{H}), 7.04(\mathrm{~d}, J=9.5 \mathrm{~Hz}, 1 \mathrm{H}), 5.88(\mathrm{~m}, 1 \mathrm{H}), 5.35(\mathrm{dd}, J=17.1 \mathrm{~Hz}, 10.2 \mathrm{~Hz}, 2 \mathrm{H}), 4.02(\mathrm{~d}$, $J=5.1 \mathrm{~Hz}, 2 \mathrm{H}), 2.94(\mathrm{~s}, 3 \mathrm{H}) .{ }^{13} \mathrm{C} \mathrm{NMR}\left(75 \mathrm{MHz}, \mathrm{CDCl}_{3}\right) \delta 148.8,136.7,136.1,130.7,127.6$, 124.0, 118.9, 117.7, 56.6, 40.1 .

$\mathrm{N}$-Benzyl-N-ethyl-2,4-dinitroaniline (3o) [46]. Yield $123 \mathrm{mg}$ (41\%). Yellow solid. Mp $72-73{ }^{\circ} \mathrm{C} .{ }^{1} \mathrm{H}$ NMR $\left(300 \mathrm{MHz}, \mathrm{CDCl}_{3}\right) \delta 8.66(\mathrm{~d}, J=2.7 \mathrm{~Hz}, 1 \mathrm{H}), 8.18(\mathrm{dd}, J=9.5 \mathrm{~Hz}, 2.7 \mathrm{~Hz}$ $1 \mathrm{H}), 7.24-7.36(\mathrm{~m}, 5 \mathrm{H}), 7.09(\mathrm{~d}, J=9.5 \mathrm{~Hz}, 1 \mathrm{H}), 4.54(\mathrm{~s}, 2 \mathrm{H}), 3.37(\mathrm{q}, J=7.1 \mathrm{~Hz}, 2 \mathrm{H}), 1.26$ $(\mathrm{t}, J=7.1 \mathrm{~Hz}, 3 \mathrm{H}) .{ }^{13} \mathrm{C}$ NMR $\left(75 \mathrm{MHz}, \mathrm{CDCl}_{3}\right) \delta 148.7,135.6,129.0,128.0,127.6,127.4,123.7$, $119.5,55.2,47.8,12.4$.

$\mathrm{N}$-(2,4-Dinitrophenyl)-N-ethyl-N-(2-phenylethyl)amine (3p). Yield $178 \mathrm{mg}(59 \%)$. Yellow solid. Mp 74-76 ${ }^{\circ} \mathrm{C}(\mathrm{EtOH}) .{ }^{1} \mathrm{H}$ NMR $\left(300 \mathrm{MHz}, \mathrm{CDCl}_{3}\right) \delta 8.62(\mathrm{~d}, J=2.8 \mathrm{~Hz}, 1 \mathrm{H}), 8.17(\mathrm{dd}$, $J=9.5 \mathrm{~Hz}, 2.8 \mathrm{~Hz}, 1 \mathrm{H}), 7.05-7.29(\mathrm{~m}, 5 \mathrm{H}), 7.03(\mathrm{~d}, J=9.5 \mathrm{~Hz}, 1 \mathrm{H}), 3.54(\mathrm{t}, J=7.4 \mathrm{~Hz}, 2 \mathrm{H})$, $3.51(\mathrm{q}, J=7.1 \mathrm{~Hz}, 2 \mathrm{H}), 3.41(\mathrm{t}, J=7.4 \mathrm{~Hz}, 2 \mathrm{H}), 1.25(\mathrm{t}, J=7.1 \mathrm{~Hz}, 3 \mathrm{H}) .{ }^{13} \mathrm{C} \mathrm{NMR}(75 \mathrm{MHz}$, $\left.\mathrm{CDCl}_{3}\right) \delta 148.0,137.9,137.8,137.0,128.8,128.7,127.5,126.9,123.8,118.8,53.4,47.2,33.7$, 12.5. HRMS (ESI): calcd for $\mathrm{C}_{16} \mathrm{H}_{18} \mathrm{~N}_{3} \mathrm{O}_{4}[\mathrm{M}+\mathrm{H}]$, 316.1292; found, 316.1288.

4-(Diethylamino)-2,3,5,6-tetrafluorobenzonitrile (4a) [47]. Yield $112 \mathrm{mg}$ (45\%). Colorless solid. Mp 47-49 ${ }^{\circ} \mathrm{C} .{ }^{1} \mathrm{H}$ NMR $\left(300 \mathrm{MHz}, \mathrm{CDCl}_{3}\right) \delta 3.41(\mathrm{qm}, J=7.1 \mathrm{~Hz}, 4 \mathrm{H}), 1.20$ $(\mathrm{t}, J=7.1 \mathrm{~Hz}, 6 \mathrm{H}) .{ }^{13} \mathrm{C}$ NMR $\left(75 \mathrm{MHz}, \mathrm{CDCl}_{3}\right) \delta 148.2(\mathrm{ddm}, J=256 \mathrm{~Hz}, 19 \mathrm{~Hz}), 140.6(\mathrm{dm}$, $J=247 \mathrm{~Hz}), 134.3(\mathrm{~m}), 108.7(\mathrm{t}, J=4 \mathrm{~Hz}), 82.5(\mathrm{t}, J=18 \mathrm{~Hz}), 46.8(\mathrm{t}, J=5 \mathrm{~Hz}), 13.6(\mathrm{t}, J=2 \mathrm{~Hz})$. ${ }^{19} \mathrm{~F}$ NMR $(282 \mathrm{MHz}, \mathrm{CDCl} 3) \delta-136.5(\mathrm{q}, J=14 \mathrm{~Hz}),-151.1(\mathrm{q}, J=14 \mathrm{~Hz})$.

N,N-Diethyl-2,3,5,6-tetrafluoropyridin-4-amine (4b) [47]. Yield $164 \mathrm{mg}$ (74\%). Colorless oil. ${ }^{1} \mathrm{H}$ NMR $\left(300 \mathrm{MHz}, \mathrm{CDCl}_{3}\right) \delta 3.44(\mathrm{q}, J=7.1 \mathrm{~Hz}, 4 \mathrm{H}), 1.24(\mathrm{tm}, J=7.1 \mathrm{~Hz}, 6 \mathrm{H}) .{ }^{13} \mathrm{C}$ NMR $\left(75 \mathrm{MHz}, \mathrm{CDCl}_{3}\right) \delta 145.2(\mathrm{ddd}, J=236 \mathrm{~Hz}, 32 \mathrm{~Hz}, 2 \mathrm{~Hz}), 139.4(\mathrm{~m}), 134.3(\mathrm{dm}, J=249 \mathrm{~Hz})$, $46.6(\mathrm{t}, J=5 \mathrm{~Hz}), 13.8 .{ }^{19} \mathrm{~F} \mathrm{NMR}\left(282 \mathrm{MHz}, \mathrm{CDCl}_{3}\right) \delta-96.7(\mathrm{~m}),-158.2(\mathrm{~m})$.

4-(Cyclohexylsulfanyl)-N,N-diethyl-3,5,6-trifluoropyridin-2-amine (4c). Yield $162 \mathrm{mg}(51 \%)$. Colorless oil. ${ }^{1} \mathrm{H}$ NMR $\left(300 \mathrm{MHz}, \mathrm{CDCl}_{3}\right) \delta 3.44(\mathrm{dq}, J=7.0 \mathrm{~Hz}, 1.7 \mathrm{~Hz}, 4 \mathrm{H}), 1.63-1.95(\mathrm{~m}$, $4 \mathrm{H}), 1.30-1.59(\mathrm{~m}, 7 \mathrm{H}), 1.17(\mathrm{t}, J=7.0 \mathrm{~Hz}, 6 \mathrm{H}) .{ }^{13} \mathrm{C} \mathrm{NMR}\left(75 \mathrm{MHz}, \mathrm{CDCl}_{3}\right) \delta 144.5(\mathrm{ddd}$, $J=247 \mathrm{~Hz}, 16 \mathrm{~Hz}, 4 \mathrm{~Hz}), 143.6(\mathrm{dm}, J=239 \mathrm{~Hz}), 141.3(\mathrm{~m}), 135.2(\mathrm{dd}, J=240 \mathrm{~Hz}, 21 \mathrm{~Hz})$, $124.9(\mathrm{ddd}, J=21 \mathrm{~Hz}, 18 \mathrm{~Hz}, 4 \mathrm{~Hz}), 46.0(\mathrm{~d}, J=3 \mathrm{~Hz}), 44.2(\mathrm{~d}, J=6 \mathrm{~Hz}), 33.5,25.8,25.5,13.6$ $(\mathrm{d}, J=1 \mathrm{~Hz}) .{ }^{19} \mathrm{~F}$ NMR $\left(282 \mathrm{MHz}, \mathrm{CDCl}_{3}\right) \delta-93.3(\mathrm{~m}),-130.7(\mathrm{~m}),-152.6(\mathrm{~m})$. HRMS (ESI): calcd for $\mathrm{C}_{15} \mathrm{H}_{23} \mathrm{~F}_{3} \mathrm{~N}_{2} \mathrm{~S}$ [M + H], 319.1450; found, 319.1462. 
N,N-Diethyl-3,5,6-trifluoro-4-(phenylsulfanyl)pyridin-2-amine (4d). Yield $203 \mathrm{mg}(65 \%)$. Colorless oil. ${ }^{1} \mathrm{H}$ NMR $\left(300 \mathrm{MHz}, \mathrm{CDCl}_{3}\right) \delta$ 7.26-7.29 (m, 2H), 7.12-7.17 (m, 3H), $3.30(\mathrm{dq}$, $J=7.1 \mathrm{~Hz}, 1.7 \mathrm{~Hz}, 4 \mathrm{H}), 1.04(\mathrm{t}, J=7.1 \mathrm{~Hz}, 6 \mathrm{H}) .{ }^{13} \mathrm{C} \mathrm{NMR}\left(75 \mathrm{MHz}, \mathrm{CDCl}_{3}\right) \delta 144.8(\mathrm{ddd}$, $J=232 \mathrm{~Hz}, 16 \mathrm{~Hz}, 3 \mathrm{~Hz}), 142.2(\mathrm{dm}, J=241 \mathrm{~Hz}), 141.3(\mathrm{~m}), 134.6(\mathrm{ddd}, J=248 \mathrm{~Hz}, 30 \mathrm{~Hz}$, $2 \mathrm{~Hz}), 133.0,132.4,130.8,127.8,125.0(\mathrm{dm}, J=22 \mathrm{~Hz}), 44.2(\mathrm{~d}, J=6 \mathrm{~Hz}), 13.6(\mathrm{~d}, J=1 \mathrm{~Hz})$. ${ }^{19} \mathrm{~F}$ NMR (282 MHz, CDCl3) $\delta-92.4(\mathrm{~m}),-130.6(\mathrm{~m}),-152.7$ (m). HRMS (ESI): calcd for $\mathrm{C}_{15} \mathrm{H}_{16} \mathrm{~F}_{3} \mathrm{~N}_{2} \mathrm{~S}[\mathrm{M}+\mathrm{H}]$, 313.0971; found, 313.0972 .

4-(1,3-Benzothiazol-2-ylsulfanyl)-N,N-diethyl-3,5,6-trifluoropyridin-2-amine (4e). Yield 207 mg (56\%). Colorless solid. Mp 87-88 ${ }^{\circ} \mathrm{C}\left(\mathrm{CCl}_{4}\right) .{ }^{1} \mathrm{H}$ NMR $\left(300 \mathrm{MHz}, \mathrm{CDCl}_{3}\right) \delta 7.89(\mathrm{~d}$, $J=8.1 \mathrm{~Hz}, 1 \mathrm{H}), 7.73(\mathrm{~d}, J=7.9 \mathrm{~Hz}, 1 \mathrm{H}), 7.41(\mathrm{~m}, 1 \mathrm{H}), 7.33(\mathrm{~m}, 1 \mathrm{H}), 3.47(\mathrm{dq}, J=7.1 \mathrm{~Hz}$, $1.8 \mathrm{~Hz}, 4 \mathrm{H}), 1.21(\mathrm{t}, J=7.1 \mathrm{~Hz}, 6 \mathrm{H}) .{ }^{13} \mathrm{C} \mathrm{NMR}\left(75 \mathrm{MHz}, \mathrm{CDCl}_{3}\right) \delta 161.7,153.2,144.9(\mathrm{ddd}$, $J=232 \mathrm{~Hz}, 16 \mathrm{~Hz}, 3 \mathrm{~Hz}), 142.6(\mathrm{dm} J=259 \mathrm{~Hz}), 141.1(\mathrm{~m}), 135.9,134.2(\mathrm{ddd}, J=253 \mathrm{~Hz}$, $32 \mathrm{~Hz}, 2 \mathrm{~Hz}), 126.5,125.2,122.4,121.0,120.2(\mathrm{dm}, J=26 \mathrm{~Hz}), 44.3(\mathrm{~d}, J=6 \mathrm{~Hz}), 13.6(\mathrm{~d}$, $J=2 \mathrm{~Hz}) .{ }^{19} \mathrm{~F} \mathrm{NMR}\left(282 \mathrm{MHz}, \mathrm{CDCl}_{3}\right) \delta-90.0(\mathrm{~m}),-126.9(\mathrm{~m}),-148.9(\mathrm{~m})$. HRMS (ESI): calcd for $\mathrm{C}_{16} \mathrm{H}_{15} \mathrm{~F}_{3} \mathrm{~N}_{3} \mathrm{~S}_{2}[\mathrm{M}+\mathrm{H}], 370.0654$; found, 370.0649 .

$\mathrm{N}, \mathrm{N}$-Diethyl-1,3-benzoxazol-2-amine (4f) [48]. Yield $179 \mathrm{mg}(87 \%)$. Colorless oil. ${ }^{1} \mathrm{H}$ $\operatorname{NMR}\left(300 \mathrm{MHz}, \mathrm{CDCl}_{3}\right) \delta 7.39(\mathrm{~d}, J=7.8 \mathrm{~Hz}, 1 \mathrm{H}), 7.27(\mathrm{~d}, J=8.1 \mathrm{~Hz}, 1 \mathrm{H}), 7.15(\mathrm{~m}, 1 \mathrm{H}), 7.03$ $(\mathrm{m}, 1 \mathrm{H}), 3.62(\mathrm{q}, J=7.0 \mathrm{~Hz}, 4 \mathrm{H}), 1.31(\mathrm{t}, J=7.0 \mathrm{~Hz}, 6 \mathrm{H}) .{ }^{13} \mathrm{C} \mathrm{NMR}\left(75 \mathrm{MHz}, \mathrm{CDCl}_{3}\right) \delta 162.1$, $148.8,143.5,123.8,120.0,115.7,108.5,42.9,13.4$.

$\mathrm{N}, \mathrm{N}$-Diethyl-5-methyl-1,3-benzoxazol-2-amine (4g) [49]. Yield $171 \mathrm{mg}(84 \%)$. Colorless oil. ${ }^{1} \mathrm{H}$ NMR $\left(300 \mathrm{MHz}, \mathrm{CDCl}_{3}\right) \delta 7.19(\mathrm{~d}, J=0.6 \mathrm{~Hz}, 1 \mathrm{H}), 7.13(\mathrm{~d}, J=8.1 \mathrm{~Hz}, 1 \mathrm{H}), 6.81(\mathrm{dd}$, $J=8.1 \mathrm{~Hz}, 0.6 \mathrm{~Hz}, 1 \mathrm{H}), 3.61(\mathrm{q}, J=7.1 \mathrm{~Hz}, 4 \mathrm{H}), 2.40(\mathrm{~s}, 3 \mathrm{H}), 1.29(\mathrm{t}, J=7.1 \mathrm{~Hz}, 6 \mathrm{H}) .{ }^{13} \mathrm{C}$ NMR (75 MHz, $\left.\mathrm{CDCl}_{3}\right) \delta 162.3,146.9,143.6,133.4,120.6,116.2,107.8,42.9,21.5,13.6$.

$\mathrm{N}, \mathrm{N}$-Diethyl-5-phenyl-1,3,4-thiadiazol-2-amine (4h) [50]. Yield $179 \mathrm{mg}$ (77\%). Colorless solid. Mp 40-42 ${ }^{\circ} \mathrm{C} .{ }^{1} \mathrm{H}$ NMR $\left(300 \mathrm{MHz}, \mathrm{CDCl}_{3}\right) \delta 7.80-7.83(\mathrm{~m}, 2 \mathrm{H}), 7.41-7.44(\mathrm{~m}, 3 \mathrm{H})$, $3.62(\mathrm{q}, J=7.1 \mathrm{~Hz}, 4 \mathrm{H}), 1.33(\mathrm{t}, J=7.0 \mathrm{~Hz}, 6 \mathrm{H}) .{ }^{13} \mathrm{C} \mathrm{NMR}\left(75 \mathrm{MHz}, \mathrm{CDCl}_{3}\right) \delta 170.6,153.2$, $131.7,129.4,129.3,127.6,46.2,11.3$.

2-Chloro- $N, N$-diethylpyrimidin-4-amine (4i) [51]. Yield $133 \mathrm{mg}(72 \%)$. Colorless oil. ${ }^{1} \mathrm{H}$ $\operatorname{NMR}\left(300 \mathrm{MHz}, \mathrm{CDCl}_{3}\right) \delta 7.90(\mathrm{~d}, J=6.2 \mathrm{~Hz}, 1 \mathrm{H}), 6.19(\mathrm{~d}, J=6.2 \mathrm{~Hz}, 1 \mathrm{H}), 3.43(\mathrm{~m}, 4 \mathrm{H}), 1.13$ $(\mathrm{t}, J=7.1 \mathrm{~Hz}, 6 \mathrm{H}) .{ }^{13} \mathrm{C}$ NMR $\left(75 \mathrm{MHz}, \mathrm{CDCl}_{3}\right) \delta 161.8,160.7,156.5,101.0,42.5,12.5$.

3-Chloro-N,N-diethylpyrazin-2-amine (4j). Yield $154 \mathrm{mg}(83 \%)$. Colorless oil. ${ }^{1} \mathrm{H}$ NMR $\left(300 \mathrm{MHz}, \mathrm{CDCl}_{3}\right) \delta 8.03(\mathrm{~d}, J=2.5 \mathrm{~Hz}, 1 \mathrm{H}), 7.73(\mathrm{~d}, J=2.5 \mathrm{~Hz}, 1 \mathrm{H}), 3.53(\mathrm{q}, J=7.1 \mathrm{~Hz}, 4 \mathrm{H})$, $1.22(\mathrm{t}, J=7.1 \mathrm{~Hz}, 6 \mathrm{H}) .{ }^{13} \mathrm{C} \mathrm{NMR}\left(75 \mathrm{MHz}, \mathrm{CDCl}_{3}\right) \delta 162.4,153.8,139.4,132.8,44.3,13.2$. HRMS (ESI): calcd for $\mathrm{C}_{8} \mathrm{H}_{13} \mathrm{ClN}_{3}[\mathrm{M}+\mathrm{H}]$, 186.0793; found, 186.0783.

$\mathrm{N}, \mathrm{N}$-Diethyl-3-phenyl-1,2,4-oxadiazol-5-amine (4k) [52]. Yield $193 \mathrm{mg}(89 \%)$. Colorless oil. ${ }^{1} \mathrm{H}$ NMR $\left(300 \mathrm{MHz}, \mathrm{CDCl}_{3}\right) \delta 8.01-8.04(\mathrm{~m}, 2 \mathrm{H}), 7.44-7.47(\mathrm{~m}, 3 \mathrm{H}), 3.58(\mathrm{q}, J=7.1 \mathrm{~Hz}$, $4 \mathrm{H}), 1.31(\mathrm{t}, J=7.0 \mathrm{~Hz}, 6 \mathrm{H}) .{ }^{13} \mathrm{C} \mathrm{NMR}\left(75 \mathrm{MHz}, \mathrm{CDCl}_{3}\right) \delta 171.0,168.7,130.5,128.5,128.0$, 127.2, 43.5, 13.3 .

$\mathrm{N}, \mathrm{N}$-Diethyl-2,6-dinitro-4-(trifluoromethyl)aniline (41) [53]. Yield $236 \mathrm{mg}$ (77\%). Yellow solid. Mp 95-96 ${ }^{\circ} \mathrm{C} .{ }^{1} \mathrm{H}$ NMR $\left(300 \mathrm{MHz}, \mathrm{CDCl}_{3}\right) \delta 8.09(\mathrm{~s}, 2 \mathrm{H}), 3.13(\mathrm{q}, J=7.0 \mathrm{~Hz}, 4 \mathrm{H}), 1.19$ $(\mathrm{t}, J=7.0 \mathrm{~Hz}, 6 \mathrm{H}) .{ }^{13} \mathrm{C} \mathrm{NMR}\left(75 \mathrm{MHz}, \mathrm{CDCl}_{3}\right) \delta 145.9,141.2,126.5,123.5(\mathrm{q}, J=271 \mathrm{~Hz})$, 122.2, 46.2, 12.7. ${ }^{19} \mathrm{~F} \mathrm{NMR}\left(282 \mathrm{MHz}, \mathrm{CDCl}_{3}\right) \delta-63.8$.

N,N-Diethyl-5-nitropyridin-2-amine (4m) [54]. Yield $193 \mathrm{mg}$ (99\%). Yellow solid. Mp $72-75{ }^{\circ} \mathrm{C} .{ }^{1} \mathrm{H}$ NMR $\left(300 \mathrm{MHz}, \mathrm{CDCl}_{3}\right) \delta 9.00(\mathrm{~d}, 1.5 \mathrm{~Hz}, 1 \mathrm{H}), 8.12(\mathrm{dd}, J=9.5 \mathrm{~Hz}, 1.5 \mathrm{~Hz}$, $1 \mathrm{H}), 6.41(\mathrm{~d}, J=9.5 \mathrm{~Hz}, 1 \mathrm{H}), 3.60(\mathrm{q}, J=7.1 \mathrm{~Hz}, 4 \mathrm{H}), 1.21(\mathrm{t}, J=7.2 \mathrm{~Hz}, 6 \mathrm{H}) .{ }^{13} \mathrm{C}$ NMR $\left(75 \mathrm{MHz}, \mathrm{CDCl}_{3}\right) \delta 159.5,146.9,134.2,132.6,104.1,43.5,12.7$.

$\mathrm{N}, \mathrm{N}$-Diethyl-3-nitropyridin-2-amine (4n) [55]. Yield $189 \mathrm{mg}$ (97\%). Orange oil. ${ }^{1} \mathrm{H}$ NMR $\left(300 \mathrm{MHz}, \mathrm{CDCl}_{3}\right) \delta 8.28(\mathrm{dd}, J=4.5 \mathrm{~Hz}, 1.7 \mathrm{~Hz}, 1 \mathrm{H}), 8.01(\mathrm{dd}, J=8.0 \mathrm{~Hz}, 1.7 \mathrm{~Hz}, 1 \mathrm{H})$, $6.63(\mathrm{dd}, J=8.1 \mathrm{~Hz}, 4.5 \mathrm{~Hz}, 1 \mathrm{H}), 3.42(\mathrm{q}, J=7.2 \mathrm{~Hz}, 4 \mathrm{H}), 1.20(\mathrm{t}, J=7.2 \mathrm{~Hz}, 6 \mathrm{H}) .{ }^{13} \mathrm{C}$ NMR $\left(75 \mathrm{MHz}, \mathrm{CDCl}_{3}\right) \delta 152.1,151.2,135.2,132.7,117.8,44.1,12.6$.

5-Bromo-N,N-diethyl-3-nitropyridin-2-amine (4o). Yield $262 \mathrm{mg}(96 \%)$. Orange oil. ${ }^{1} \mathrm{H}$ $\operatorname{NMR}\left(300 \mathrm{MHz}, \mathrm{CDCl}_{3}\right) \delta 8.31(\mathrm{~d}, J=2.2 \mathrm{~Hz}, 1 \mathrm{H}), 8.15(\mathrm{~d}, J=2.2 \mathrm{~Hz}, 1 \mathrm{H}), 3.42(\mathrm{q}, J=7.1 \mathrm{~Hz}$, $4 \mathrm{H}), 1.20(\mathrm{t}, J=7.1 \mathrm{~Hz}, 6 \mathrm{H}) .{ }^{13} \mathrm{C} \mathrm{NMR}\left(75 \mathrm{MHz}, \mathrm{CDCl}_{3}\right) \delta 151.9,150.6,136.8,104.3,44.3$, 12.5. HRMS (ESI): calcd for $\mathrm{C}_{9} \mathrm{H}_{13} \mathrm{BrN}_{3} \mathrm{O}_{2}$ [M + H], 274.0186; found, 274.0194 . 
N,N-Diethyl-3-(1-methylethyl)-1,2,4-oxadiazol-5-amine (4p). Yield $118 \mathrm{mg}$ (86\%). Colorless oil. ${ }^{1} \mathrm{H}$ NMR $\left(300 \mathrm{MHz} \mathrm{CDCl}_{3}\right) \delta 3.43(\mathrm{q}, J=7.1 \mathrm{~Hz}, 4 \mathrm{H}), 2.82(\mathrm{~h}, J=7.0 \mathrm{~Hz}, 1 \mathrm{H}), 1.23$ $(\mathrm{d}, J=7.0 \mathrm{~Hz}, 6 \mathrm{H}), 1.19(\mathrm{t}, J=7.1 \mathrm{~Hz}, 6 \mathrm{H}) .{ }^{13} \mathrm{C} \mathrm{NMR}\left(75 \mathrm{MHz}, \mathrm{CDCl}_{3}\right) \delta 175.6,170.6,43.3$, 27.0, 20.4, 13.2. HRMS (ESI): calcd for $\mathrm{C}_{9} \mathrm{H}_{18} \mathrm{~N}_{2} \mathrm{O}[\mathrm{M}+\mathrm{H}], 184.1444$; found, 184.1451 .

\subsubsection{Mechanistic Experiment}

$f a c-\operatorname{Ir}(\text { ppy })_{3}(2 \mathrm{mg}, 0.003 \mathrm{mmol})$ and $N$-methylpyrrolidine $(170 \mathrm{mg}, 2 \mathrm{mmol})$ were added to a solution of pentafluoropyridine $(169 \mathrm{mg}, 1 \mathrm{mmol})$ in acetonitrile $(2 \mathrm{~mL})$. The mixture was irradiated at room temperature with blue LED for $20 \mathrm{~h}$. An aliquot was withdrawn and analyzed by ${ }^{1} \mathrm{H},{ }^{13} \mathrm{C},{ }^{19} \mathrm{~F}$ NMR. The solvent was evaporated under vacuum, the residue was washed with methyl tert-butyl ether $(4 \times 5 \mathrm{~mL})$. The obtained solid was dried under vacuum and dissolved in DMSO- $\mathrm{d}_{6}$. NMR analysis indicated the presence of N,N-dimethylpyrrolidinium salt. ${ }^{1} \mathrm{H}$ NMR (300 MHz, DMSO-d 6 ) $\delta 3.47(\mathrm{~m}, 4 \mathrm{H}), 3.11$ (s, 6H), $2.10(\mathrm{~m}, 4 \mathrm{H}) .{ }^{13} \mathrm{C}$ NMR $\left(75 \mathrm{MHz}, \mathrm{DMSO}_{-} \mathrm{d}_{6}\right) \delta 65.3,51.6,21.8 .{ }^{19} \mathrm{~F} \mathrm{NMR}(282 \mathrm{MHz}$, DMSO-d 6 ) $\delta-138.4$ (br).

\section{Conclusions}

A method for the dealkylative arylation of tertiary amines by means of electron deficient aromatic and heteroaromatic halides under photoredox conditions is described. The reaction proceeds through the generation of aryl radicals, which interact with the amines followed by the dealkylation of the tryalkylamino fragment.

Supplementary Materials: The following are available online, Figures S1-S4: Cyclic voltammetry curves; Cartesian coordinates and energies; Copies of NMR spectra.

Author Contributions: D.L.L., A.E.F., A.Y.T., experiment; V.V.L., calculations and writing; A.D.D., writing. All authors have read and agreed to the published version of the manuscript.

Funding: This work was supported by Scientific Schools Development Program by Zelinsky Institute of organic chemistry.

Institutional Review Board Statement: Not applicable.

Informed Consent Statement: Not applicable.

Data Availability Statement: The data presented in this study are available in this article.

Acknowledgments: The authors thank Vladimir Kokorekin (Zelinsky Institute) for performing cyclic voltammetry measurements.

Conflicts of Interest: The authors declare no conflict of interest.

Sample Availability: Not applicable.

\section{References}

1. Terrier, F. Modern Nucleophilic Aromatic Substitution; Wiley-VCH: Weinheim, Germany, 2013.

2. Caron, S.; McInturff, E. Nucleophilic aromatic substitution. In Practical Synthetic Organic Chemistry; Caron, S., Ed.; John Wiley \& Sons Inc.: Hoboken, NJ, USA, 2020; pp. 231-246.

3. Sandford, G. Perfluoroheteroaromatic chemistry: Multifunctional systems from perfluorinated heterocycles by nucleophilic aromatic substitution processes. Top. Heterocycl. Chem. 2012, 27, 1-32.

4. Langlois, B.; Gilbert, L.; Forat, G.; Jean-Roger, D.; Serge, R. Fluorination of aromatic compounds by halogen exchange with fluoride anions ("halex" reaction). In Industrial Chemistry Library; Desmurs, J.-R., Ratton, S., Eds.; Elsevier: Amsterdam, The Netherlands, 1996; Volume 8, pp. 244-292.

5. Hartwig, J.F. Transition metal catalyzed synthesis of arylamines and aryl ethers from aryl halides and triflates: Scope and mechanism. Angew. Chem. Int. Ed. 1998, 37, 2047-2067. [CrossRef]

6. Yang, B.H.; Buchwald, S.L. Palladium-catalyzed amination of aryl halides and sulfonates. J. Organomet. Chem. 1999, 576, 125-146. [CrossRef]

7. Buchwald, S.L.; Mauger, C.; Mignani, G.; Scholz, U. Industrial-scale palladium-catalyzed coupling of aryl halides and amines-A personal account. Adv. Synth. Catal. 2006, 348, 23-39. [CrossRef]

8. Sambiagio, C.; Marsden, S.P.; Blacker, A.J.; McGowan, P.C. Copper catalysed Ullmann type chemistry: From mechanistic aspects to modern development. Chem. Soc. Rev. 2014, 43, 3525-3550. [CrossRef] [PubMed] 
9. Walsh, K.; Sneddon, H.F.; Moody, C.J. Amination of heteroaryl chlorides: Palladium catalysis or $\mathrm{S}_{\mathrm{N}}$ Ar in green solvents? ChemSusChem 2013, 6, 1455-1460. [CrossRef]

10. Prier, C.K.; Rankic, D.A.; MacMillan, D.W.C. Visible light photoredox catalysis with transition metal complexes: Applications in organic synthesis. Chem. Rev. 2013, 113, 5322-5363. [CrossRef] [PubMed]

11. Marzo, L.; Pagire, S.K.; Reiser, O.; König, B. Visible-light photocatalysis: Does it make a difference in organic synthesis? Angew. Chem. Int. Ed. 2018, 57, 10034-10072. [CrossRef]

12. Majek, M.; Jacobi von Wangelin, A. Mechanistic perspectives on organic photoredox catalysis for aromatic substitutions. Acc. Chem. Res. 2016, 49, 2316-2327. [CrossRef]

13. Tay, N.E.S.; Nicewicz, D.A. Cation radical accelerated nucleophilic aromatic substitution via organic photoredox catalysis. J. Am. Chem. Soc. 2017, 139, 16100-16104. [CrossRef]

14. Pistritto, V.A.; Schutzbach-Horton, M.E.; Nicewicz, D.A. Nucleophilic aromatic substitution of unactivated fluoroarenes enabled by organic photoredox catalysis. J. Am. Chem. Soc. 2020, 142, 17187-17194. [CrossRef] [PubMed]

15. Venditto, N.J.; Nicewicz, D.A. Cation radical-accelerated nucleophilic aromatic substitution for amination of alkoxyarenes. Org. Lett. 2020, 22, 4817-4822. [CrossRef] [PubMed]

16. Holmberg-Douglas, N.; Nicewicz, D.A. Arene cyanation via cation-radical accelerated-nucleophilic aromatic substitution. Org. Lett. 2019, 21, 7114-7118. [CrossRef]

17. Tay, N.E.S.; Chen, W.; Levens, A.; Pistritto, V.A.; Huang, Z.; Wu, Z.; Li, Z.; Nicewicz, D.A. ${ }^{19}$ F- and ${ }^{18}$ F-arene deoxyfluorination via organic photoredox-catalysed polarity-reversed nucleophilic aromatic substitution. Nat. Catal. 2020, 3, 734-742. [CrossRef] [PubMed]

18. Liu, W.; Li, J.; Huang, C.-Y.; Li, C.-J. Aromatic chemistry in the excited state: Facilitating metal-free substitutions and crosscouplings. Angew. Chem. Int. Ed. 2020, 59, 1786-1796. [CrossRef] [PubMed]

19. Cowper, N.G.W.; Chernowsky, C.P.; Williams, O.P.; Wickens, Z.K. Potent reductants via electron-primed photoredox catalysis: Unlocking aryl chlorides for radical coupling. J. Am. Chem. Soc. 2020, 142, 2093-2099. [CrossRef]

20. Corcoran, E.B.; Pirnot, M.T.; Lin, S.; Dreher, S.D.; DiRocco, D.A.; Davies, I.W.; Buchwald, S.L.; MacMillan, D.W.C. Aryl amination using ligand-free $\mathrm{Ni}$ (II) salts and photoredox catalysis. Science 2016, 353, 279-283. [CrossRef]

21. Zuo, Z.; Cong, H.; Li, W.; Choi, J.; Fu, G.C.; MacMillan, D.W.C. Enantioselective decarboxylative arylation of o \pm -amino acids via the merger of photoredox and nickel catalysis. J. Am. Chem. Soc. 2016, 138, 1832-1835. [CrossRef]

22. Lavagnino, M.N.; Liang, T.; MacMillan, D.W.C. HARC as an open-shell strategy to bypass oxidative addition in UllmannGoldberg couplings. Proc. Natl. Acad. Sci. USA 2020, 117, 21058-21064. [CrossRef]

23. Kim, K.; Hong, S.H. Photoinduced Copper(I)-catalyzed cyanation of aromatic halides at room temperature. Adv. Synth. Catal. 2017, 359, 2345-2351. [CrossRef]

24. Arora, A.; Weaver, J.D. Visible Light photocatalysis for the generation and use of reactive azolyl and polyfluoroaryl intermediates. Acc. Chem. Res. 2016, 49, 2273-2283. [CrossRef] [PubMed]

25. Singh, A.; Fennell, C.J.; Weaver, J.D. Photocatalyst size controls electron and energy transfer: Selectable $E / Z$ isomer synthesis via C-F alkenylation. Chem. Sci. 2016, 7, 6796-6802. [CrossRef]

26. Meyer, A.U.; Slanina, T.; Yao, C.-J.; Koenig, B. Metal-free perfluoroarylation by visible light photoredox catalysis. ACS Catal. 2016, 6, 369-375. [CrossRef]

27. Senaweera, S.; Weaver, J.D. Dual C-F, C-H Functionalization via photocatalysis: Access to multifluorinated biaryls. J. Am. Chem. Soc. 2016, 138, 2520-2523. [CrossRef] [PubMed]

28. Ou, W.; Zou, R.; Han, M.; Yu, L.; Su, C. Tailorable carbazolyl cyanobenzene-based photocatalysts for visible light-induced reduction of aryl halides. Chin. Chem. Lett. 2020, 31, 1899-1902. [CrossRef]

29. Hamilton, G.L.; Backes, B.J. Dealkylative functionalization of tertiary amines with electron deficient heteroaryl chlorides. Tetrahedron Lett. 2006, 47, 2229-2231. [CrossRef]

30. Khalaf, A.I.; Alvarez, R.G.; Suckling, C.J.; Waigh, R.D. Unexpected dealkylation during nucleophilic substitution: Synthesis of 2-N,N-dialkylamino benzoxazoles and benzothiazoles. Tetrahedron 2000, 56, 8567-8571. [CrossRef]

31. Kolesinska, B.; Kaminski, Z.J. The umpolung of substituent effect in nucleophilic aromatic substitution. A new approach to the synthesis of $\mathrm{N}, \mathrm{N}$-disubstituted melamines (triazine triskelions) under mild reaction conditions. Tetrahedron 2009, 65, 3573-3576. [CrossRef]

32. Lee, M.; Rucil, T.; Hesek, D.; Oliver, A.G.; Fisher, J.F.; Mobashery, S. Regioselective control of the $\mathrm{S}_{\mathrm{N}}$ Ar amination of 5-substituted2,4-dichloropyrimidines using tertiary amine nucleophiles. J. Org. Chem. 2015, 80, 7757-7763. [CrossRef]

33. Zubkov, M.O.; Kosobokov, M.D.; Levin, V.V.; Kokorekin, V.A.; Korlyukov, A.A.; Hu, J.; Dilman, A.D. A novel photoredox-active group for the generation of fluorinated radicals from difluorostyrenes. Chem. Sci. 2020, 11, 737-741. [CrossRef]

34. Panferova, L.I.; Zubkov, M.O.; Kokorekin, V.A.; Levin, V.V.; Dilman, A.D. Using the thiyl radical for aliphatic hydrogen-atom transfer: Thiolation of unactivated C-H bonds. Angew. Chem. Int. Ed. 2021, 60, 2849-2854. [CrossRef] [PubMed]

35. Ormazabal-Toledo, R.; Richter, S.; Robles-Navarro, A.; Maulen, B.; Matute, R.A.; Gallardo-Fuentes, S. Meisenheimer complexes as hidden intermediates in the aza-S $\mathrm{S}_{\mathrm{N}}$ Ar mechanism. Org. Biomol. Chem. 2020, 18, 4238-4247. [CrossRef]

36. Christopher, J.A.; Brophy, L.; Lynn, S.M.; Miller, D.D.; Sloan, L.A.; Sandford, G. Synthetic utility of 4-bromo-2,3,5,6tetrafluoropyridine. J. Fluorine Chem. 2008, 129, 447-454. [CrossRef] 
37. Grossi, L.; Strazzari, S. Aromatic radical anions as possible intermediates in the nucleophilic aromatic substitution $\left(\mathrm{S}_{\mathrm{N}} \mathrm{Ar}\right)$ : An EPR study. J. Chem. Soc. Perkin Trans. 2 1999, 2141-2146. [CrossRef]

38. Imoto, M.; Matsui, Y.; Takeda, M.; Tamaki, A.; Taniguchi, H.; Mizuno, K.; Ikeda, H. A probable hydrogen-bonded meisenheimer complex: An unusually high $\mathrm{S}_{\mathrm{N}}$ Ar reactivity of nitroaniline derivatives with hydroxide ion in aqueous media. J. Org. Chem. 2011, 76, 6356-6361. [CrossRef]

39. Kuehne, M.E. The arylation of enamines. J. Am. Chem. Soc. 1962, 84, 837-847. [CrossRef]

40. Panahi, F.; Daneshgar, F.; Haghighi, F.; Khalafi-Nezhad, A. Immobilized Pd nanoparticles on silica-starch substrate (PNP-SSS): Efficient heterogeneous catalyst in Buchwald-Hartwig C-N cross coupling reaction. J. Organomet. Chem. 2017, 851, $210-217$. [CrossRef]

41. Al-Rawi, J.M.A.; Khuthier, A.-H.; Hanna, S.Y. Carbon-13 nuclear magnetic resonance spectra of some N-(2,4-dinitrophenyl) Amines, The Corresponding N-Oxides, and Their Thermal Rearrangement Products. Spectrosc. Lett. 1988, 21, 249-259. [CrossRef]

42. Feng, Y.-S.; Mao, L.; Bu, X.-S.; Dai, J.-J.; Xu, H.-J. Pd(OAc) 2 -catalyzed dinitration reaction of aromatic amines. Tetrahedron 2015, 71, 3827-3832. [CrossRef]

43. Bellucci, G. Synthesis and determination of the configuration of the diastereoisomeric 4,N-dimethyl- $N$-phenylcyclohexylamines. Gazz. Chim. Ital. 1969, 99, 1208-1216.

44. Graymore, J. 175. The reduction products of certain cyclic methyleneamines. Part II. J. Chem. Soc. 1932, 1353-1357. [CrossRef]

45. Kleinschmidt, R.F.; Cope, A.C. Rearrangement of allyl groups in dyad systems. amine oxides. J. Am. Chem. Soc. 1944, 66, 1929-1933. [CrossRef]

46. McIntire, F.C.; Clements, L.M.; Sproull, M. 1-Fluoro-2,4-dinitrobenzene as quantitative reagent for primary and secondary amines. Anal. Chem. 1953, 25, 1757-1758. [CrossRef]

47. Matsuda, N.; Hirano, K.; Satoh, T.; Miura, M. Copper-catalyzed direct amination of polyfluoroarenes and azoles with hydroxylamines and its application to the synthesis of 3-aminobenzoheteroles. Synthesis 2012, 44, 1792-1797. [CrossRef]

48. Grube, H.; Suhr, H. Nucleophile substitution, XI. reaktionen von chlorsubstituierten heteroaromaten mit aminen. Chem. Ber. 1969, 102, 1570-1579. [CrossRef]

49. Qiu, Y.; Struwe, J.; Meyer, T.H.; Oliveira, J.C.A.; Ackermann, L. Catalyst- and reagent-free electrochemical azole C-H amination. Chem. Eur. J. 2018, 24, 12784-12789. [CrossRef] [PubMed]

50. Yonemoto, K.; Shibuya, I. Reaction of 1,4,2-dithiazolium salts with amino compounds. Bull. Chem. Soc. Jpn. 1988, 61, 4043-4049. [CrossRef]

51. Jo, J.; Kim, S.H.; Kim, H.; Jeong, M.; Kwak, J.-H.; Taek Han, Y.; Jeong, J.-Y.; Jung, Y.-S.; Yun, H. Discovery and SAR studies of novel 2-anilinopyrimidine-based selective inhibitors against triple-negative breast cancer cell line MDA-MB-468. Bioorg. Med. Chem. Lett. 2019, 29, 62-65. [CrossRef] [PubMed]

52. Su, D.; Duan, H.; Wei, Z.; Cao, J.; Liang, D.; Lin, Y. Condensation of Vilsmeier salts, derived from tetraalkylureas, with amidoximes: A novel approach to access N,N-dialkyl-1,2,4-oxadiazol-5-amines. Tetrahedron Lett. 2013, 54, 6959-6963. [CrossRef]

53. Joardar, S.; Bhattacharyya, A.; Das, S. A Palladium on carbon catalyzed one-pot synthesis of substituted benzimidazoles. Synthesis 2014, 46, 3121-3132. [CrossRef]

54. Adamson, A.J.; Jondi, W.J.; Tipping, A.E. Reaction of metal diethylnitroxides with pentafluoropyridine, pentafluorobenzene, octafluorotoluene and 2-chloro-3- or 5-nitropyridine. J. Fluorine Chem. 1996, 76, 67-78. [CrossRef]

55. Nandi, D.; Islam, R.U.; Devi, N.; Siwal, S.; Mallick, K. A palladium nanoparticle-catalyzed aryl-amine coupling reaction: High performance of aryl and pyridyl chlorides as the coupling partner. New J. Chem. 2018, 42, 812-816. [CrossRef] 